\title{
Educar en el humanismo solidario. Los retos para la educación universitaria católica
}

\author{
Educating in solidary humanism. The challenge for Catholic \\ university education
}

Laura Noemí Urbina Valor ${ }^{1}$

Citar: Urbina Valor, L. N. (2020). Educar en el humanismo solidario. Los retos para la educación universitaria católica. Cuadernos Universitarios, 13, pp. 37-48.

Recibido: setiembre 2020

Aceptado: octubre 2020

Ensayo científico

\section{Resumen}

Este trabajo se propone realizar una reflexión acerca de los retos de la universidad católica para cumplir su actual misión educativa a partir de los aportes que efectuó al mundo la encíclica Populorum Progressio, con el concepto de desarrollo humano integral y solidario, y de los lineamientos propuestos en Educar al humanismo solidario. Para construir una «civilización del amor».50 años después de la Populorum progressio (Congregación para la Educación Católica, 2017). El reto que se presenta a la universidad católica es poner en el centro de la educación la relación con la persona concreta y real a través de sus tres funciones sustantivas. Se propone para ello trabajar con líneas de reflexión prioritarias, promover la unidad de los conocimientos, el trabajo interdisciplinario y el diálogo constante con la cultura y las ciencias, generando así un proyecto ético integral universitario y un modelo pedagógico como el de la pedagogía de la alteridad, entre otros.

Palabras clave: desarrollo humano integral - humanismo solidario - educación universitaria católica - alteridad - reflexión

\footnotetext{
${ }^{1}$ Universidad Católica de Salta (UCASAL). Profesora de Filosofía y Licenciada en Psicopedagogía. Se desempeña como jefa del Departamento de Filosofía y Ética del Vicerrectorado de Formación. Es profesora de Ética Profesional y de Responsabilidad Social en carreras de la UCASAL. Ha integrado equipos de investigación del área de la ética y ha dictado cursos en relación a esa disciplina y a la responsabilidad social empresarial. Se desempeñó en comisiones como la de Ética Aplicada y la de Responsabilidad Social Universitaria.1urbina@ucasal.edu.ar
} 


\section{Abstract}

This work aims to make a reflection about the Catholic university challenges in order to fulfill its current educational mission based on the contributions made to the world by the encyclical Populorum progressio, with the concept of integral human development and solidarity, and the guidelines proposed in Educating for a Humanism of Solidarity. To Build a «Civilization of Love». 50 Years After Populorum progressio (Congregation for Catholic Education, 2017). The challenge presented to the Catholic university is to place at the center of education the relationship with the concrete and real person through its three substantive functions. To this end, it proposes to work with priority lines of reflection, promote unity of knowledge, interdisciplinary work and constant dialogue with culture and science, thus generating an integral ethical university project and a pedagogical model such as the pedagogy of otherness, among others.

Key words: integral human development Humanism of Solidarity - Catholic university education - otherness - reflection

\section{Introducción}

Este trabajo se propone realizar una reflexión acerca de los retos de la universidad católica para cumplir su misión educativa en la actualidad; misión expresada en la carta apostólica Ex Corde Ecclesiae (Juan Pablo II, 1990) sobre las universidades católicas.

Con este propósito, se consideran los valiosos aportes que la encíclica Populorum progressio efectuó al mundo, con el concepto de desarrollo humano integral y solidario, de todo el hombre y de todos los hombres; concepto de gran fecundidad a pesar de las variaciones de las condiciones culturales, socioeconómicas y políticas y en el desarrollo del conocimiento científico-técnico ocurridas en el mundo desde el momento de su publicación.

Se consideran, además, los lineamientos para la educación propuestas en el documento Educar al humanismo solidario. Para construir una «civilización del amon».50 años después de la Populorum progressio (Congregación para la Educación Católica [CEC], 2017), que moviliza a las instituciones educativas católicas en la generación de respuestas en el contexto actual.

Con estas premisas, se pretende reflexionar sobre las cuestiones implicadas en la búsque- da de caminos para realizar la misión educativa encomendada por la Iglesia a las universidades católicas, buscando a partir de la antropología cristiana los caminos formativos que permitan dar respuestas al hombre concreto, en las coordenadas actuales, a fin de trazar claras líneas de acción y un proyecto educativo consensuado, integral, ético, basado en la escucha sincera de la voz del otro; pero con firmes bases en la identidad que le es propia.

\section{Desarrollo humano integral y solidario}

La carta encíclica Populorum progressio, del papa Pablo VI, promulgada el 26 de marzo de 1967, llamaba la atención sobre el carácter mundial que había adquirido la cuestión social, haciendo un llamado a favorecer el desarrollo integral del hombre y el desarrollo solidario de la humanidad a través de acciones concretas.

E1 desarrollo puede ser entendido de diferentes modos. Entonces, cabe la pregunta: ¿cuál es el concepto de desarrollo propuesto en la encíclica? De hecho, no se trata de un concepto basado en lo económico, enfoque tan difundido en la época en que fue escrito este documento. Sino que se trata de aquel que tiene por centro al hombre, que busca que el 
hombre sea más hombre y que reconoce que «hacer, conocer, y tener más para ser más: tal es la aspiración de los hombres de hoy» (p. 6). Y, por ello, sostiene que el verdadero desarrollo consiste en «el paso para cada uno y para todos, de condiciones de vida menos humanas a condiciones más humanas» (p. 20). Destaca, además, otro aspecto no menos importante y es que no existe plenitud humana sin Dios como horizonte de sentido, porque la persona está llamada a la trascendencia, a lograr su plenitud en Dios. De allí que en la Populorum progressio se integran la visión humana, ya que el centro es el ser humano, y la visión cristiana, porque no hay verdadera plenitud sin Dios.

Por lo tanto, en la encíclica se puede notar que el desarrollo, para que sea auténtico, necesariamente tiene que ser integral, «de todo el hombre y de todos los hombres» (p. 42). Constituye una vocación, porque es un llamado que proviene de lo profundo del ser humano, pues existen en él potencialidades que debe hacer crecer, fructificar, como ser libre y responsable. A su vez, solo es posible que se realice a sí mismo superándose; por lo que ese desarrollo tiene que estar abierto a Dios como su fin último, para el logro de la plenitud humana.

Esas son las bases para que se pueda hablar de desarrollo humano integral, auténtico, y este es el sólido fundamento sobre el cual se puede desplegar el desarrollo solidario de la humanidad. Este es el gran aporte que realiza la encíclica a la reflexión ética de su tiempo y de la posteridad.

\section{Líneas centrales de la propuesta educativa de la Iglesia}

Considerando estos conceptos tan importantes de la Populorum progressio, en septiembre de 2017, la Congregación para la Educación Católica presentó en el Vaticano el documento:
Educar al humanismo solidario. Para construir una «civilización del amor». 50 años después de la Populorum progressio.

En su introducción, señala que nos encontramos ante un proceso de cambio epocal, en el que se pone en evidencia un humanismo decadente, el cual con frecuencia se basa en el paradigma de la indiferencia. Resalta, por ello, la necesidad de que el humanismo solidario sea realmente vivido y testimoniado.

Sostiene que, en estas nuevas condiciones que nos toca vivir, toman especial relevancia las palabras de Benedicto XVI en la carta encíclica Caritas in veritate, respecto a que hoy la cuestión social se torna en una cuestión antropológica; por lo que la educación en estos tiempos tiene un papel fundamental e impostergable.

A partir de estas premisas, se plantean en el documento las líneas centrales de la propuesta educativa de la Iglesia: la humanización de la educación, la cultura del diálogo, la globalización de la esperanza, el logro de una verdadera inclusión y la construcción de redes de cooperación.

Pero ¿qué significa humanizar la educación? Significa poner a la persona en el centro de la educación, considerando que educar es un proceso a través del cual esta puede desarrollar sus actitudes y vocación, pero en un marco de relaciones dentro de una comunidad viva, unida a un destino común, de modo tal que le permita contribuir a la vocación de la propia comunidad. Significa, entre otras cosas, que la educación tiene que estar al servicio del cuerpo social, que ofrece lugares de encuentro y confrontación, que produce comunión y experiencias de compartir.

En un mundo caracterizado por la multiculturalidad, el documento expresa que se necesita una cultura del diálogo. Es decir, que el diálogo se lleve a cabo en un marco ético, de 
libertad e igualdad, con una actitud formativa, con reconocimiento de la dignidad de los interlocutores y con la intención de construir puentes en la búsqueda de respuestas. En ese sentido, el humanismo solidario tiene un papel importante en la formación de ciudadanos dialogantes, en la actitud de encuentro en las diferencias, en un marco de valores que esté presente en los programas formativos.

No obstante, la misión propia de la educación al humanismo solidario es la globalización de la esperanza. En efecto, se trata de una tarea específica de la educación cristiana, porque se sitúa en la dinámica de hacer crecer, de dar la vida, germen de esperanza. Es preciso tener en cuenta, en este sentido, que el hombre puede ser redimido por el amor; no por la ciencia. Pero las ciencias, informadas por la caridad, pueden ayudar al hombre a la búsqueda de sentido. Por eso la necesidad de que la enseñanza de las ciencias sea efectuada con vistas a la "conciencia de un universo ético".

Precisamente, en el seno de las relaciones educativas, se tiene que enseñar el amor cristiano y es a partir de allí donde se puede desarrollar el bien común e impulsar no solo la realización personal, sino también la del género humano.

Globalizar la esperanza es considerar las posibilidades que esta trae, pero también la exclusión a que puede llevar; por eso es necesaria una globalización con visión y esperanza.

Otra de las grandes metas de la educación es la inclusión. En este sentido, el documento advierte que hoy conocemos, por el progreso científico y técnico, que las decisiones que se toman no solo afectan la forma de vida actual, sino también la existencia de las personas del futuro (CEC, 2017, p. 21). Se precisa, entonces, expandir los límites de solidaridad y asumir una perspectiva más amplia respecto al bien común, a partir de una concepción ética del universo.
Esto plantea una tarea importante a la educación basada en el humanismo solidario, que consiste en favorecer la construcción de una cultura fundada en una ética intergeneracional; y, a la vez, un reto especial para las universidades, ya que a través de sus programas académicos se tendrían que desarrollar las competencias necesarias para la toma de decisiones en relación con la sostenibilidad y con las necesidades de las futuras generaciones.

La inclusión presenta otro reto a la educación, que consiste en considerar la solidaridad con las generaciones precedentes. Ello demanda la formación de una conciencia histórica, que rescate la conciencia de unión entre las diferentes generaciones y considere que la forma en que nuestros antepasados superaron sus dificultades y desafíos también puede servir para el presente.

La última meta que subraya la carta es en relación con las redes de cooperación. Se plantea la necesidad de generar dinámicas incluyentes y la socialización del humanismo solidario a través de redes de cooperación, donde los actores educativos desarrollen actitudes colaborativas. Hay un llamado especial en relación con las universidades, a generar investigaciones colectivas en diferentes áreas del conocimiento, a desarrollar investigaciones que involucran docentes, jóvenes investigadores y estudiantes y redes de cooperación con otras áreas no educativas. Esta colaboración también tendría que darse entre las diferentes instituciones académicas nacionales e internacionales, sea de investigación o de servicio.

\section{Retos de la de la universidad católica para cumplir su misión educativa}

Ahora bien, lo expresado por la Congregación para la Educación Católica plantea desafíos insoslayables a las instituciones educativas 
católicas para educar en el humanismo solidario, en un mundo atravesado por cambios sociales, culturales, políticos, económicos, medioambientales, y en donde incluso se discute la idea de lo humano.

La universidad católica «mantiene con la Iglesia una vinculación, que es esencial para su identidad institucional» (Ex Corde Ecclesiae [ECE], n. $\left.{ }^{\circ} 27\right)$. Y la «misión fundamental de la universidad es la constante búsqueda de la verdad mediante la investigación, la conservación y la comunicación del saber para el bien de la sociedad» (ECE, n. $\left.{ }^{\circ} 30\right)$.

De acuerdo con la Ex Corde Ecclesiae, la finalidad del quehacer de las instituciones universitarias católicas es lograr una presencia pública, continua y universal del pensamiento cristiano en todo esfuerzo tendiente a promover la cultura superior.

La universidad católica está llamada a asumir los grandes retos para cumplir con su misión específica y para poder aportar al mundo actual a partir de una educación basada en la perspectiva del humanismo integral y solidario, en la construcción de la «civilización del amor».

La pregunta que surge, entonces, es ¿ cómo llevar a cabo el humanismo integral y solidario desde la universidad? ¿De qué modo esta podría contribuir al logro de un desarrollo humano integral, armónico, orientado, que responda a las necesidades sin generar desequilibrios?

Si partimos de una visión de la universidad con una perspectiva basada en un fuerte compromiso con el desarrollo humano - entendido como desarrollo integral y solidario— una cuestión a clarificar como comunidad es cuál es el futuro que se desea para la humanidad, para poder proyectar y orientar una educación posible.

En relación a esto último, es interesante rescatar el pensamiento del filósofo Jacques Maritain, quien destaca la necesidad de ser consecuentes con las nuevas condiciones históricas, conservando los principios de una civilización que sea vitalmente cristiana, pero traducida a las nuevas condiciones. De acuerdo con ello, el humanismo de hoy tendría que centrarse en la «rehabilitación de la criatura en Dios» (Maritain, 1999, p. 104)².

Partimos, entonces, del reconocimiento que existen en el mundo transformaciones que alcanzan diversas dimensiones y que exigen análisis y clarificación. Entre ellas se encuentran los cambios en las identidades individuales y sociales, producidos por las tecnologías del cuerpo y del yo, las desestructuraciones culturales y las biotecnologías, las nuevas ideas acerca del cosmos, la naturaleza y los límites (o su ausencia) del conocimiento científico y de la tecnología.

Los procesos de cambio presentan también aspectos ambiguos, como la tendencia a la mundialización y el resurgimiento de nacionalismos y racismos, el crecimiento económico y la marginación social. Nos encontramos con la lógica de un modelo economicista (un orden mundial basado en un sistema económico mundial), un modelo de desarrollo único, un proceso de globalización tecnológico y cultural, que privilegia comportamientos como la posesión, la acumulación, el individualismo, con el consiguiente empo-

\footnotetext{
${ }^{2}$ Idelfonso Camacho explica, en su artículo «Populorum progressio: desarrollo integral y humanismo cristiano», que para Maritain la construcción de la ciudad temporal no constituye una obra divina que el hombre tiene que realizar en la Tierra, sino que se trata de una obra humana a realizar en la Tierra con sus propios medios y su trabajo, bajo la inspiración del amor. Este es el humanismo integral o teocéntrico, cuyas características son opuestas al liberalismo o al humanismo antropocéntrico.
} 
brecimiento de la existencia y la subvaluación de las dimensiones afectivas, estéticas, éticas y las espirituales en general.

Hoy también es posible conocer muchas de las consecuencias positivas o negativas que el obrar humano podría tener para la humanidad presente y las generaciones futuras. Todo esto requiere repensar la realidad, ya sea en sus valores e ideas, como en las repercusiones que estas tienen en los aspectos sociales, económicos, políticos, y medioambientales de diferente signo que están presentes e influyen en la forma de vida de todos los pueblos.

En los momentos actuales de la humanidad, las relaciones con el otro se ven signadas por sentimientos y experiencias de hostilidad, por el aislamiento y la soledad y el debilitamiento de vínculos reales y desinteresados. Aparecen los resentimientos y la desconfianza entre grupos, las rivalidades y agresiones y la dificultad de las relaciones interculturales.

Por ello, la universidad como comunidad «en salida» tiene un quehacer impostergable: promover la reflexión sobre las grandes cuestiones que enfrenta la humanidad «atreviéndose a llegar a todas las periferias que necesitan la luz del Evangelio» (Evangelii gaudium, n. ${ }^{\circ}$ 20), en un diálogo permanente con la realidad; pues, como institución formadora, es el ámbito privilegiado para generar las propuestas que reclama el mundo actual, que ayuden al hombre a entender esa realidad y a saber responder a los retos que esta le plantea, contribuyendo a su crecimiento hacia una responsabilidad común por el bien de todos.

Especialmente en las universidades católicas se hace indispensable considerar la existencia de la tensión dialéctica entre cultura y cristianismo. Por tal motivo, será necesario promover un diálogo fecundo entre fe y cultura, entre revelación y problemas humanos; ya que esto permitirá que se generen respuestas que sean respetuosas y coherentes con la dignidad humana y que estén en sintonía con los valores del Evangelio.

Se precisa entonces que, desde las instituciones académicas, se trabaje por una cultura de la solidaridad, que contribuya a una vida más digna, coadyuvando a la eliminación de la pobreza y de los problemas humanos que ella acarrea; de manera comprometida en la consecución de una mayor justicia social, luchando para cambiar los sistemas sociales basados en el egoísmo y no en el altruismo; denunciando aquellas lógicas de justificación con las que se intenta legitimar las desigualdades sociales.

Otro de los grandes desafíos a afrontar es, justamente, la diversidad de información, de opiniones y de posiciones que ofrecen las culturas y las nuevas tecnologías.

La universidad deberá marcar rumbos, mostrar caminos posibles en ese «aprender a ser»; porque le toca actuar en un mundo marcado por la tendencia al «tener más», donde se privilegia frecuentemente una visión materialista y fragmentada de la realidad y que anula el concepto de verdad absoluta y universal - supuesto posmoderno-, sosteniendo que cualquier mirada de la realidad posee el mismo crédito. Tendrá que evitar toda forma de deshumanización, en una cultura con grandes contradicciones, donde a menudo surgen posiciones que, bajo la apariencia de la defensa de derechos, tienden a desvalorizar al ser humano, tratándolo como un objeto.

\section{Líneas o propuestas de acción posibles}

Una de las formas que se propone para que la universidad católica cumpla con esta tarea es generar «líneas de reflexión prioritarias» que atraviesen sus funciones propias, estableciendo un diálogo constante y fecundo 
con la cultura y las ciencias; considerando la necesidad de ir formando un liderazgo intelectual en esos ámbitos.

Las líneas definidas institucionalmente como ejes, como fruto de la reflexión conjunta, deberían referirse a cuestiones prioritarias y que no son posibles de soslayar; tales como la relación de la ciencia y la tecnología con la vida humana, la degradación del medio ambiente natural y humano, la manipulación genética, las problemáticas de la vida social, de índole local, regional o humana, en general, entre otras. El objeto sería promover la reflexión, la investigación y la acción, así como un modo de pensar y de sentir que implique disposición para el encuentro con el otro, la tolerancia, la empatía; denunciando aquellas actitudes contrarias a la consideración de la condición humana y a una convivencia armoniosa.

Estas líneas de reflexión prioritarias tendrían que ser incorporadas y estar presentes tanto en los programas de estudio de la formación de grado y posgrado como en los proyectos de tesis, en jornadas y diferentes propuestas académicas, en distintos foros y ámbitos de discusión, ya sea hacia dentro de la universidad como hacia la comunidad. Y, además, tendrían que trabajarse en los proyectos de investigación, preferentemente interdisciplinarios, cuyos resultados generen conocimiento y abran propuestas. Sería preciso que estos involucren a docentes y alumnos, dando lugar a redes cooperativas, en las que participen miembros de diferentes universidades, nacionales e internacionales. Asimismo, sería preciso que se convocara a participar en proyectos compartidos a distintos sectores de la comunidad, para buscar respuestas en un marco ético a las profundas cuestiones que atraviesan a la sociedad, con los parámetros del respeto, la justicia, la solidaridad y la responsabilidad en relación a las actuales y futuras generaciones.
Frente a la diversidad de información, de opiniones y de posiciones que presentan las nuevas tecnologías, se torna imprescindible que se habiliten ciertos espacios de diálogo y discusión que posibiliten una manera de acercarse al conocimiento, una visión de la realidad, una forma de construir saberes y actitudes, de producir y transformar; que permitan e impulsen la generación de proyectos individuales y colectivos que surjan en el seno la universidad o que sean promovidos por ella.

Pero también es necesario lograr la integración del saber, pues se tiene que tener en claro que «repensar la educación significa, ante todo, promover la unidad de los conocimientos como antídoto a la fragmentación y al panorama sociocultural desintegrado» (Zani, 2019). La integración del saber no consiste en sostener una posición ecléctica. Lejos de ello, implica que la comunidad universitaria asuma la búsqueda de la unidad del conocimiento y de la verdad, haciéndose presente en sus funciones sustantivas. Por eso, también es importante propiciar el diálogo fe-razón, considerando la «inculturación de la fe y la evangelización de las culturas» (Versaldi, 2017, p. 19).

Desde la función de la docencia — sin excluir las otras funciones-, una de las tareas primordiales será «enseñar a pensar», para formar personas autónomas, con sentido crítico y conciencia social, que puedan tomar decisiones prudentes y manifestar coherencia entre el pensamiento y la acción, para así ser capaces de contribuir a la transformación creativa de la sociedad. Esto significa, entre otras cosas, enseñar a asumir la defensa de la dignidad de la persona y su desarrollo y a practicar dicha defensa con acciones específicas.

En esa línea, una preocupación primordial de la universidad para avanzar en humanidad, en este hacerse más humano, sería generar tanto una especial sensibilidad hacia la situa- 
ción personal del ser humano concreto, considerando sus sufrimientos físicos y morales, sus sentimientos, ilusiones y esperanzas, como también una sensibilidad para identificar tendencias sociales y de cualquier índole que atenten contra el bien de las personas. La incorporación de un centro de asesoría psicológica o de conversatorios sobre temas específicos son algunas de las acciones posibles en este ámbito, además del trabajo de la pastoral universitaria y de la coordinación de espiritualidad.

Pensar la formación universitaria implica no solo pensar un plan de estudios, la elaboración de la currícula, sino también considerar qué tipo de relación tendría que establecerse entre docente y alumno para que se pueda hacer efectiva la enseñanza. Enseñar es «un acto de transmisión cultural con intenciones sociales y opciones de valor» (Davini, 2008, p. 17); donde quien enseña trata de transmitir un saber, una práctica considerada culturalmente válida, que ayude al desarrollo de las personas, a la formación de buenos ciudadanos y que sea valiosa desde un punto de vista ético. En este sentido, siempre la enseñanza responde a intenciones y es una acción conscientemente dirigida para que alguien aprenda algo. Por ello, no pueden entrar en contradicción los propósitos explícitos del docente y el modo en que enseña.

Tiene especial significación, desde esta perspectiva, el diálogo con los jóvenes estudiantes, que no consiste solo en un intercambio de ideas sino también en un «intercambio de dones» (Juan Pablo II, 1995), donde el docente es capaz de unir a la verdad que enseña «el testimonio de su vida, en íntima relación con el saber que enseña» (Bergoglio, 2008). Y, como expresa la Congregación para la Educación Católica, «los profesores tienen muchas responsabilidades y su compromiso debe transformarse cada vez más en una acción real, creativa e inclusiva» (CEC, 2020).

Por lo dicho, y puesto que educar es transmitir esperanzas, afectos, convicciones, es preciso que todos los integrantes de la comunidad universitaria estén involucrados en esta tarea. Y el mensaje de esperanza tiene que ser transmitido tanto por la razón como por la acción, apoyado por la autenticidad y la coherencia en la propia vida. No es posible la neutralidad, se requieren convicción y un compromiso personal profundo con estos valores.

Justamente, en relación con este aspecto, la reciente Carta circular a las escuelas, universidades e instituciones educativas, de la Congregación para la Educación Católica, expresa que «las relaciones interpersonales constituyen el 'lugar' donde la transdisciplinariedad y la interdisciplinariedad emergen como criterios culturales fundamentales para frenar los riesgos de fragmentación y desintegración del conocimiento» $y$, también, el lugar de «la apertura de este mismo conocimiento a la luz de la Revelación» (CEC, 2020).

Es digna de considerar también la necesaria construcción de un «modelo pedagógico», que contribuya al diseño de intencionalidades educativas con base en la pregunta por los fines constitutivos del fenómeno educativo, para responder mejor a las exigencias éticas originarias de la educación. Si consideramos que «toda actividad educativa, ya sea explícita o más veladamente, se orienta hacia unos fines concretos que tienen que ver con un determinado modelo de hombre y de sociedad al que se pretende aspirar» (Mínguez, Romero y Pedreño, 2016, pp. 164-165), se requiere hacer explícita esa concepción de base.

Ese modelo pedagógico podría basarse, por ejemplo, en el aporte de la filosofía de Emma- 
nuel Levinas, que permite la reflexión sobre una «ética de la educación» ${ }^{3}$. Levinas sostiene que lo que define al hombre no es el saber sino el amor a los demás. Desde su postura, realiza una crítica al humanismo occidental, proponiendo el humanismo del otro, que se fundamenta en la responsabilidad absoluta por el otro. Así, el otro tiene una primacía monumental ante el yo (mismo), teniendo presente que la subjetividad se construye desde la alteridad, como ser-para-el-otro; y lo que llamamos «rostro» es el modo por el que se me presenta el otro y «el acceso al rostro es, de entrada, ético» (Levinas, 2000, p. 71). El planteo filosófico de Levinas ha dado lugar a una «pedagogía de la alteridad», que centra su reflexión en la comprensión del ser humano y en la idea de alteridad, en la experiencia y el testimonio del educador, constituyéndose en una pedagogía testimonial donde «nada está establecido de antemano» (Ortega, 2016, p. 259).

Especial valor posee el saber ético, para este tipo de educación, como orientación y guía de la responsabilidad personal y del vivir social, que se nutre de la esencia ética individual y de la comunidad académica. Solo una genuina instancia ética puede favorecer la individuación de la verdadera felicidad hacia la cual cada persona tiende, sostenida por una sólida vida espiritual con compromiso comunitario.

Será preciso, entonces, buscar la consolidación en la universidad de un "proyecto ético integral», que abarque todos los componentes de la comunidad educativa y en relación con la sociedad, impulsando conductas que sean coherentes con el ideario de la universidad, basado en un profundo respeto por la digni- dad humana y en una cultura de la solidaridad. Este proyecto debería orientar todos los procesos decisorios que se tomen, las soluciones que se propongan y los retos que encare la universidad.

El desarrollo humano integral y solidario exige a la universidad la tarea de la preservación de los valores de las culturas locales, a través de la promoción y el fortalecimiento de las diferentes expresiones culturales, ofreciendo diversas oportunidades formativas, suscitando el encuentro, la convivencia y el intercambio. En este sentido, se requiere bregar por el cuidado del medioambiente, tan amenazado en la época actual; porque como dice el Papa Francisco, «el ambiente natural y el ambiente humano se degradan juntos, y no podremos afrontar adecuadamente la degradación ambiental si no prestamos atención a causas que tienen que ver con la degradación humana y social» (papa Francisco, 2015, p. 48). Es preciso entonces enfrentar el deterioro de la «casa común», levantando la voz para hacerse escuchar y encontrar las vías para evitar daños y solucionar problemas, en búsqueda de la «conversión ecológica».

También la universidad debe trabajar por el reconocimiento del valor del patrimonio natural e histórico-cultural (material e inmaterial), en el que se generan y activan nexos de identidad, frente al sincretismo cultural producido por la globalización; en el rescate del saber y de las experiencias de un pueblo, que pueden resultar enriquecedoras y aportar soluciones a las cuestiones y realidades que lo afligen. Así, se puede actualizar el pacto educativo entre generaciones.

\footnotetext{
${ }^{3}$ Las ideas de Levinas dieron lugar al planteo de una pedagogía de la alteridad, una de cuyas direcciones asume la cuestión del otro como eje ético fundamental en el proceso educativo, donde la relación educador-educando se funda en la acogida y la responsabilidad común como base de una educación en valores en relación con el contexto en que se inscribe.
} 
Por último, para influir en la cultura son importantes además otro tipo de acciones concretas: la participación en los ámbitos donde se toman decisiones, se diseñan planes o se elaboran leyes y en las instancias de generación de corrientes de opinión.

Estas son algunas propuestas para enfrentar los desafíos que le caben a la universidad católica, en esa búsqueda del desarrollo humano integral y solidario, de la armonía del ser humano con el universo y su apertura a Dios creador.

\section{Conclusiones}

En este trabajo se presentaron algunos esbozos acerca de formas posibles en que podrían encauzarse estos desafíos de educar al humanismo solidario. Somos conscientes de que el reto de la universidad católica en el mundo de hoy reviste complejidad; sin embargo, tiene que asumir el reto de educar al humanismo solidario, poniendo en el centro de la educación la relación con la persona concreta y real, basándose en una visión a la vez humana y cristiana de la realidad.

Esto significa que, desde sus funciones sustantivas, la universidad trate de:

- Generar en la universidad «líneas de reflexión prioritarias» para propiciar la consideración de puntos de vista, argumentaciones y propuestas que alimenten la reflexión individual y que promuevan el debate colectivo y la investigación sobre problemáticas actuales en la búsqueda de lograr una transformación de aquellas realidades que lo ameritan. Se considera necesario que esas líneas de reflexión prioritarias atraviesen las funciones sustantivas de la universidad, a la vez que se conformen redes de cooperación entre integrantes de la comunidad universitaria, y con otras universidades y sectores de la sociedad.

- Asumir un diálogo con las ciencias y con las diversas culturas, considerando los principios del cristianismo, pero aplicados a las nuevas condiciones que nos tocan en estos tiempos y en la búsqueda de una trascendencia que no sea solo horizontal, que termine en una inmanencia en el mismo horizonte humano, sino también de una trascendencia vertical, en una apertura a Dios.

- Promover la reflexión, la toma de conciencia y la responsabilidad personal y comunitaria, en la construcción de una convivencia y un destino compartido; por lo que es importante lograr un «liderazgo intelectual».

- Bregar por la integración del saber, a través de la superación de visiones individualistas y estrechas de la realidad, frente a la visión fragmentaria del saber, basada a su vez en una visión fragmentaria de la realidad. En este sentido, se propone el trabajo interdisciplinario en las tres funciones de la universidad y, a la vez, promover en la comunidad universitaria y en su contexto una actitud de diálogo para la solución de los problemas más acuciantes, desarrollando compromiso con la comunidad y el contexto local. Esto sin dejar de generar también una especial sensibilidad hacia la situación personal del ser humano concreto, para lo que sería conveniente la creación de un centro de asesoría psicológica, de conversatorios sobre temas específicos, además de las acciones de la pastoral universitaria o de la coordinación de espiritualidad.

- Promover la «jerarquización de la ética» como eje formativo en todo programa de 
estudio, pero además en las propias prácticas universitarias, atravesando todas las dimensiones de la universidad; ya que esta proporciona el modelo o guía de conducta para el desarrollo de valores personales y sociales. Por eso es importante ir avanzando en un proyecto ético integral universitario, para fijar valores rectores en la toma de decisiones en la universidad.

- Suscitar un modelo pedagógico que considere central las relaciones educador-educando, el encuentro con el otro, enraizado en sus circunstancias socio-históricas; ya que posibilita el crecimiento de las personas en su relación con las demás en forma comunitaria. Por eso se propone la pedagogía de la alteridad, que centra su reflexión en la comprensión del ser humano y en la idea de alteridad, en la experiencia y el testimonio del educador.

- Preservar los valores de las culturas locales, cuidando el ambiente natural y humano, rescatando los saberes y experiencias de un pueblo, actualizando el pacto educativo intergeneracional.

- Impulsar acciones a través de la participación en los ámbitos donde se toman decisiones, se diseñan planes o se elaboran leyes y en las instancias de generación de corrientes de opinión.

Queda mucho aún por considerar. Pero lo fundamental para poder promover un auténtico humanismo, un humanismo integral, trascendente y abierto a Dios, es tener presente como comunidad educativa y como institución académica que no se puede avanzar en un verdadero proyecto de ese tipo sin el esclarecimiento acerca de qué desarrollo se quiere lograr, sin un planteo profundo acerca del tipo de sociedad que se desea construir y al servicio de qué tipo de persona, para luego conducir las fuerzas institucionales en ese sentido.

\section{Referencias bibliográficas}

Benedicto XVI (2009). Caritas in veritate. (Carta encíclica). Disponible en: http:// www.vatican.va/content/benedict-xvi/ es/encyclicals/documents/hf_ben-xvi_ enc_20090629_caritas-in-veritate.html

Bergoglio, M. (2008). Mensaje durante la misa por la educación. Buenos Aires. Disponible en: https://www.arzbaires.org.ar/inicio/homilias/homilias2008.htm\# educacion

Camacho, I. (2017). «Populorum progressio: desarrollo integral y humanismo cristiano». Veritas, (37), 123-148. Recuperado de: https://dx.doi.org/10.4067/S071892732017000200123.

Congregación para la Educación Católica (2017). Educar al humanismo solidario. Para construir una «civilización del amor». 50 años después de la Populorum progressio. Disponible en: http:/www.vatican.va/roman_curia/congregations/ccatheduc/documents/rc_con_ccatheduc_doc_20170416_ educare-umanesimo-solidale_sp.html

Congregación para la Educación Católica (2020). Carta circular a las escuelas, universidades e instituciones educativas. Disponible en: https://www.ucsf.edu.ar/ carta-circular-a-las-escuelas-universidades-e-instituciones-educativas/

Davini, M. C. (2008) Métodos de enseñanza. Didáctica general para maestros y profesores. Buenos Aires: Santillana.

Juan Pablo II (1990). Constitución apostólica Ex Corde Ecclesiae (Desde el corazón de la Iglesia). Disponible en: http://www.vatican. va/content/john-paul-ii/es/apost_constitu- 
tions/documents/hf_jp-ii_apc_15081990_ ex-corde-ecclesiae.html

Lévinas, E. (2000). Ética e Infinito. Madrid: Machado Libros.

Maritain, J. (1999). Humanismo integral. Problemas temporales y espirituales de una nueva cristiandad. Madrid: Palabra.

Mínguez, R.; Romero, E. y Pedreño, M. (2016). «La pedagogía del otro: bases antropológicas e implicaciones educativas». Teoría de la Educación. Revista Interuniversitaria. 28(2), 163-183.

Ortega, P. (2016). «La ética de la compasión en la pedagogía de la alteridad». Revista Española de Pedagogía, 74(264), 243-264.

Papa Francisco (2013). Exhortación apostólica Evangelii gaudium del santo padre Francisco a los obispos, a los presbiteros y diáconos. A las personas consagradas y a los fieles laicos sobre el anuncio del Evangelio en el mundo actual. Disponible en: http:// www.vatican.va/content/dam/francesco/ pdf/apost_exhortations/documents/papafrancesco_esortazione-ap_20131124_ evangelii-gaudium_sp.pdf

Papa Francisco (2015). Laudato si'. Sobre el cuidado de la casa común. (Carta encíclica). Disponible en: https://www.oas.org/es/ $\mathrm{sg} /$ casacomun/docs/papa-francesco-enciclica-laudato-si-sp.pdf

Versaldi, G. (2017). La educación católica en una sociedad pluralista. Buenos Aires: Ágape.

Zani, A. V. (8 de junio de 2019). Discurso de clausura. Congreso Mundial OIEC. Nueva York, Estados Unidos. Disponible en: http://www.educatio.va/content/dam/cec/ Documenti/AR-OIEC-NEW-YORK\%20 